\title{
Effect of Water Clover (Marsilea crenata) Ethanol Extracts on Follicle and Oocyte Diameter of Goat: In Vitro Study
}

\author{
Siska Nanda Widhaningrum ${ }^{1}$, Septiawan Putranto ${ }^{1}$, Sri Rahayu ${ }^{2 *}$, Gatot Ciptadi ${ }^{3}$ \\ ${ }^{1}$ Master Program of Biology, Faculty of Mathematics and Natural Sciences, University of Brawijaya, Malang, Indonesia \\ 2Department of Biology, Faculty of Mathematics and Natural Sciences, University of Brawijaya, Malang, Indonesia \\ ${ }^{3}$ Faculty of Animal Husbandry, University of Brawijaya, Malang, Indonesia
}

\begin{abstract}
Water clover (Marsilea crenata) is one of the herbal plants that has been using in alternative medicine. It possesses pharmacologically active compounds like flavonoid, which has cellular activities such as antioxidant and estrogenic activity. This study aimed to evaluate the impact of water clover ethanol extract (WCE) at different concentrations on the growth of follicles and oocytes based on follicles and oocytes diameter, respectively, after six days of culture. This experimental study used 24 isolated antral follicles $(2.5-3.2 \mathrm{~mm})$, which were randomly divided into four groups including control (without supplemented WCE) and experimental groups that supplemented with different concentrations of $\operatorname{WCE}\left(21.6,43.2\right.$, and $\left.86.4 \mathrm{~g} \mathrm{~m} \mathrm{~m}^{-1}\right)$ in culture medium for six days culture. The diameter of follicles was measured on days 0,3 , and 6 . Additionally, oocytes diameter was also measured on day 6 . The results indicate that the mean diameter of antral follicles and oocyte diameter of WCE $43.2 \mu \mathrm{g} \mathrm{ml}^{-1}$ was significantly increase compared to the other groups $(\mathrm{P} \leq 0.05)$. According to our results, WCE exerts its effect on the growth of the antral follicle and oocyte based on follicles and oocytes diameter respectively in a dose-dependent manner after six days of the antral follicle cultured.
\end{abstract}

Keywords: antral follicle, flavonoid, in vitro, oocyte, water clover.

\section{INTRODUCTION}

Reproduction is a critical biological process and very important in all living systems to maintain species survival [1]. The ovarian follicle is the structural and functional unit of the female reproductive system, which plays a role in folliculogenesis [2]. Folliculogenesis is the physiological process that involves a complex interaction among endocrine, paracrine, and autocrine factors for activation, growth, and maturation of the ovarian follicles. So, it influences steroidogenesis, angiogenesis, oocyte maturation, as well as follicular atresia [3].

During folliculogenesis, reactive oxygen species (ROS) will be generated as a result of cellular metabolism. Cell living under aerobic need oxygen, so ROS such as superoxide anion radical $\left(\mathrm{O}^{-}\right)$, hydroxyl radical $\left(\mathrm{OH}^{-}\right)$, and hydrogen peroxide $\left(\mathrm{H}_{2} \mathrm{O}_{2}\right)$ will be generated from oxygen [4]. Also, superoxide anion radical (O2) reacts with nitric oxide (NO-) to form peroxynitrite $\left(\mathrm{ONOO}^{-}\right)$, so it will form reactive nitrogen species (RNS) [5]. The increase of ROS and RNS levels react with cellular lipids, protein, and nucleic acid, so that lead to significant damage of cell structure and thereby cause

*Correspondence address:

Sri Rahayu

Email : yayuksrirahayu8@gmail.com

Address : Dept. Biology, University of Brawijaya, Veteran Malang, Malang 65145 oxidative stress (OS). Several studies indicate that OS is involved in the initiation of apoptosis in antral follicles, so that affect of folliculogenesis and steroidogenesis [6]. However, the role of endogen antioxidants such as superoxide dismutase (SOD), glutathione peroxidase, and catalase reduced the increase of free radical and maintain the proper redox state of cells as the signalling molecules in normal physiological processes [5].

Folliculogenesis includes the growth and maturation of follicle which provides the microenvironment necessary for oocyte growth and development, so it will produce matured oocyte (metaphase II) [7]. The growth of follicles is characterized by increasing in follicular diameter due to an increase in oocytes diameter, the number of granulosa cells layer, and formation of the antrum (accumulation of follicular fluid) [8]. Oocyte quality can be determined by the oocyte growth based on oocyte diameter [9].

Water clover (Marsilea crenata) is one of the herbal plants that has been using in alternative medicine for osteoporosis, infection of the urinary tract, and inflammation of the esophagus [10]. It is caused by the phytochemical content of flavonoids in WCE, which have cellular activities such as antioxidant, anti-inflammatory, anti- tumor, anti-osteoporosis, and estrogenic [11]. The previous study explained that the 
presence of flavonoids as an antioxidant in semen extender can improve the quality of semen from the effects of freezing [12]. Besides, isoflavone, the subclass of flavonoid also exerts estrogenic activity that plays an important role in folliculogenesis [13]. Therefore, the present study aimed to evaluate the impact of water clover ethanol extract (WCE) at the different concentrations on the growth of follicles and oocytes based on the follicles and oocytes diameter respectively, after the six days of the antral follicle cultured.

\section{MATERIAL AND METHOD Extraction of Marcilea crenata}

The leaves of WCE were collected in Surabaya, East Java, Indonesia. Leaves were washed thoroughly with water then dried in the greenhouse at room temperature $\left(25^{\circ} \mathrm{C}\right)$ during 4-5 days. The dried leaves were ground into powder [14] then extracted with ethanol $70 \%$ through the maceration method with modification [15]. Ethanol extraction of phenolic compounds in WCE was carried out at the Biochemistry Laboratory, Faculty of Mathematics and Natural Sciences, Brawijaya University. The next step was freeze-dried for 24 hours to get the extracts in the form of pasta.

\section{Isolated Antral Follicles and IVGC}

This study used the antral follicle of Etawah Crossbred (PE) to analyze the effect of WCE on folliculogenesis. Goat ovaries were collected from slaughterhouse Malang City, East Java, Indonesia. The ovaries were washed with sterile $0.9 \%$ physiological saline and then were transported to the laboratory in a container kept at $37^{\circ} \mathrm{C}$ in sterile $0.9 \%$ physiological saline supplemented with antibiotics, which consist of $0.01 \mathrm{~g}$ streptomycin and $0.006 \mathrm{~g}$ penicillin (Meiji, Japan). After arrived at the laboratory, some fat tissues were removed from ovaries and washed again in sterile $0.9 \%$ physiological saline [16].

The antral follicles with diameter size of 2.5$3.2 \mathrm{~mm}(\mathrm{n}=24)$ were isolated with the slicing technique and cultured for 6 days in $300 \mu \mathrm{L}$ of culture medium in each well. The culture medium containing TCM-199 medium supplemented with $10 \%$ heat-activated Fetal Bovine Serum (FBS), 1\% antibiotic Pen-Strep, 0.1 IU. $\mathrm{mL}^{-1} \mathrm{FSH}, 1$ IU. $\mathrm{mL}^{-1}$ HCG, 4\% polyvinylpyrrolidone, then covered under sterilized paraffin oil and incubation at $37^{\circ} \mathrm{C}$ in $5 \% \mathrm{CO}_{2}$ [17]. WCE were added in the treated groups at 21.6, 43.2, and $86.4 \mu \mathrm{g} \cdot \mathrm{mL}^{-1}$. And then, half of the culture medium (approximately $150 \mu \mathrm{L}$ ) was replaced every day, except the culture medium of the third day that was completely replaced.

\section{Morphological Evaluation of Follicular Growth}

Evaluation of the growth of antral follicles morphology was checked by stereo-microscope (SMZ 645 Nikon, Tokyo, Japan) that have been approved with a camera at $\times 20$ magnification and observed on days 0,3 , and 6 . And then measured of follicular diameter were carried out by Image raster software [18].

\section{Evaluation of Oocyte Quality}

Evaluation of oocyte quality used oocyte diameter was carried out on the sixth day of culture. The oocytes in selected antral follicles were isolated with the slicing technique and then measured of oocyte diameter by an inverted microscope at $x 10$ magnification [8].

\section{Statistical Analysis}

The data were presented in mean values and Standard Error (SE). The data were analyzed by one-way ANOVA using SPSS 16 for windows. If the result of ANOVA Test shows significantly different $(P \leq 0.05)$ and followed by Posthoc Test using Duncan with a confident level of $95 \%$ $(\alpha=0.05)$.

\section{RESULT AND DISCUSSION}

Effect of WCE on the diameter of cultured isolated antral follicles

One of the purposes of this study was to evaluate the impact of different concentrations of WCE on the growth of isolated cultured antral follicles for six days. The mean diameter in the sixth day cultured of the isolated cultured antral follicles, which were treated by WCE $43.2 \mu \mathrm{g} \cdot \mathrm{mL}^{-1}$ was significantly increase compared to the other groups (Table 1, Fig. 1).

During folliculogenesis, the increasing of follicular diameter occurred due to the influence of gonadotropins, such as FSH promotes proliferation and differentiation of granulosa cell through cAMP-PKA and PI3K-AKT pathway [19]. Thus, the follicle able to form an antrum (follicular fluids were produced by the granulosa cell), and the follicular diameter will increase. In addition, 17 $\beta$-estradiol through sex steroidogenesis also promotes proliferation and differentiation of granulosa cells in the presence of FSH stimulation [20]. The differentiation of granulosa cells can be divided into 2 groups, such as cumulus cells surrounding the oocyte to promote maturation oocyte and mural granulosa 
cell to produce sex steroid. So, the increase in the number of granulosa cells, oocyte diameter, and antrum formation cause the increase of follicular diameter [8].

Tabel 1. The average follicles diameter during in vitro culture

\begin{tabular}{|c|c|c|c|}
\hline \multirow{2}{*}{ Groups } & \multicolumn{3}{|c|}{ Diameter of Follicles $(\mu \mathrm{m})$} \\
\hline & Day-0 & Day-3 & Day-6 \\
\hline \multirow{2}{*}{ Control } & $2927.30 \pm$ & $3105.06 \pm$ & $3023.75 \pm$ \\
\hline & 160.29 & $241.44^{*}$ & 120.77 \\
\hline \multirow{2}{*}{$\mathrm{D} 21.6 \mu \mathrm{g} \cdot \mathrm{ml}^{-1}$} & $2889.77 \pm$ & $2770.85 \pm$ & $2879.03 \pm$ \\
\hline & 184.05 & 260.75 & 205.02 \\
\hline \multirow{2}{*}{$\mathrm{D} 43.2 \mu \mathrm{g} . \mathrm{ml}^{-1}$} & $2873.25 \pm$ & $3106.83 \pm$ & $3462.75 \pm$ \\
\hline & 82.83 & $210.63^{*}$ & $341.26^{*}$ \\
\hline \multirow{2}{*}{$\mathrm{D} 86.4 \mu \mathrm{g} \cdot \mathrm{ml}^{-1}$} & $2838.10 \pm$ & $2781.14 \pm$ & $2879.77 \pm$ \\
\hline & 214.68 & 300.51 & 245.14 \\
\hline
\end{tabular}

Notes: symbol $(*)$ indicate a significant difference between treatment $(p<0.05)$.

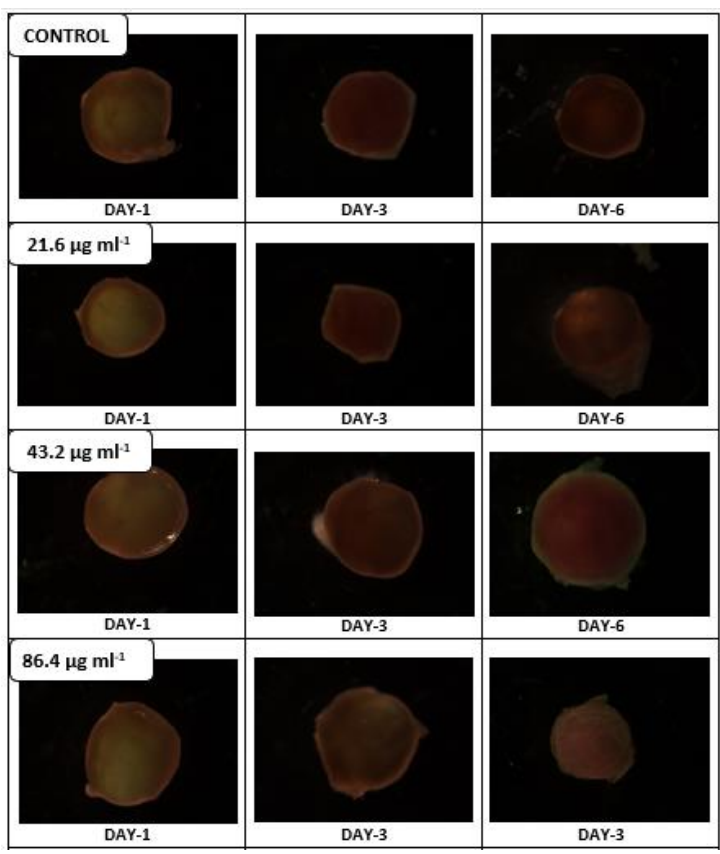

Figure 1. Isolated antral follicles in vitro culture in different treatments: the control group (first row), this experimental group supplemented with 21.6, 43.2, and $86.4 \mu \mathrm{g} \cdot \mathrm{mL}^{-1}$ (second, third, and fourth row respectively) on day 0 , day 3 , and day 6 . Images were observed by the stereo microscope that has been approved with a camera at $\times 20$ magnification

The increased follicular diameter after WCE treatment may occurred by antioxidant activity in WCE with binding to free radicals through the phenolic hydroxyl structure of flavonoid [21]. So, it can inhibit cell damage due to the increase of free radicals and can maintain the level of intracellular homeostasis between prooxidant and antioxidant [5]. In addition, flavonoids also regulate the activity of the antioxidant enzyme to inhibit cell damage and followed by apoptosis [22]. In addition, OS causes a decrease in follicular diameter, which can initiate apoptosis in antral follicles and affect follicular development and steroidogenesis [5]. Overproduction of ROS can impact the IVC of antral follicles because they act as the second messenger and induce the opening of a nonspecific pore in the inner mitochondria membrane. Thus, it releases cytochrome $C$ and is followed by caspase activation to induce apoptosis and loss of follicular function [23].

The growth of the antral follicle also occurred due to the presence of phytoestrogens in WCE that produce estrogenic activity/agonist. So, there was a bind between phytoestrogen and estrogen receptor, such as ER $\alpha$ and ER $\beta$ in cytoplasm or nucleus through genomic and nongenomic action with lower binding compared to endogenous estrogen $17 \beta$-estradiol [24]. This complex yields estrogenic activity functioned as transcription factors that initiate the transcription activity of a gene [19-21]. This complex also yields a rapid cellular response, such as MAPK/ERK, PI3K/AKT, CAMP/PKA, PKC, tyrosine kinase pathway, which is also be used to increase transcription activity [25].

According to Rosales-Tores [20], the presence of estrogenic activity may increase the response of granulosa cells to FSH stimulation. It enables the increasing proliferation and differentiation activity by expressing several genes that play important role in the folliculogenesis of antral follicles, such as FSH (FSHR), luteinizing hormone receptor (LHR), aromatase (CYP19a1), cytochrome P450 (CYP11a1), 17 $\alpha$-hydroxylase

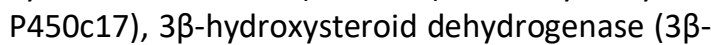
HSD).

Whereas, the decreasing of follicular diameter occurred due to the phytoestrogens at certain dosage enable to yield antiestrogenic activity/antagonist, so they unable to promote proliferation and differentiation in follicle development of antral follicle. Based on the previous study, phytoestrogens at high doses inhibit the activity of cytochrome p450 (CYP11a1), which can convert cholesterol into pregnenolone, thus affect estrogen production [26]. Genistein, one of the phytoestrogens also alters the level of precursor hormones of estrogen, such as estrone, testoserone, DHEA, progresterone, and enables cause follicle atresia 
that followed by reduction of oocyte maturation. Besides, genistein also significantly increases the expression of the cell cycle inhibitor (cyclindependent kinase inhibitor $1 a / c d k n 1 a)$, thus it can inhibit proliferation of granulosa cells [27].

\section{Effect of WCE on the diameter of oocytes}

The presence of WCE in the culture medium enables to increase oocyte maturation characterized by an increase of oocyte diameter at WCE $43.2 \mu \mathrm{g} \cdot \mathrm{mL}^{-1}$ compared to the other groups (Fig. 2 and 3). The increasing oocyte diameter may occur due to antioxidant activity in WCE. So, it can suppress free radical activity in oocytes. However, overproduction of ROS exceeds physiological limit, so it can induce destabilization of maturation promoting factor (MPF), decrease survival factor, and stimulate apoptosis in oocyte through mitochondria [28].

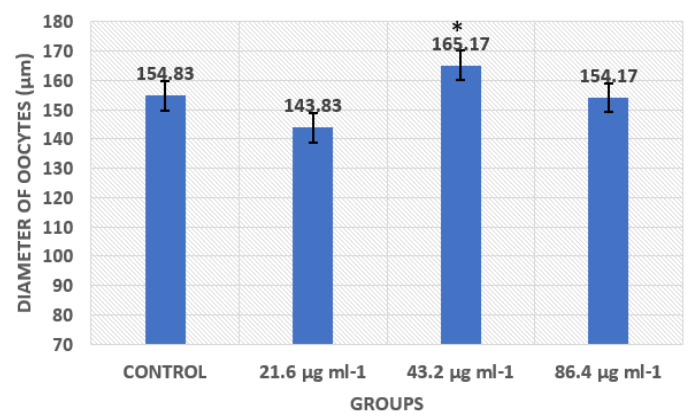

Figure 2. The average diameter of oocytes after antral follicle culture in different treatments. Symbol $(*)$ indicate a significant difference between treatment $(p<0.05)$.

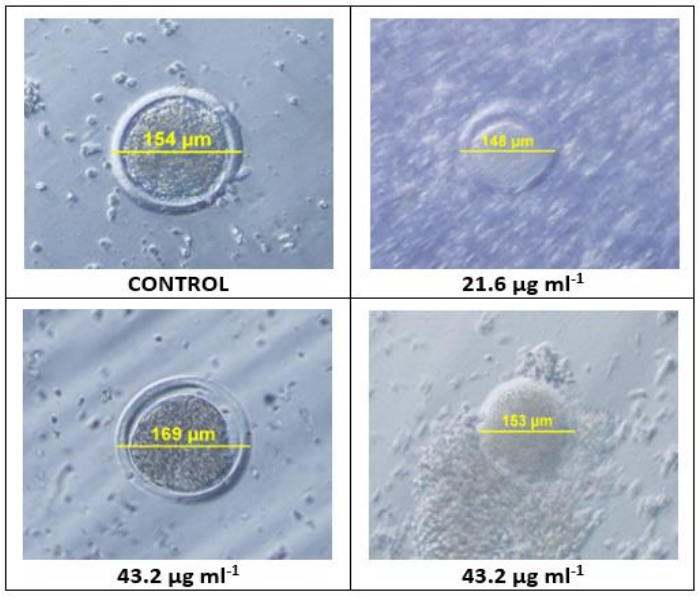

Figure 3. The diameter of oocytes after antral follicle culture in different treatments.

The decreasing of oocyte diameter occurred due to the phytoestrogens at high doses enable to inhibit the kinase activity of cyclin-dependent kinase 2 (cdk2)/cell division cycle 2 (cdc2) [29]. Thus, induce $\mathrm{G} 2 / \mathrm{M}$ arrest in mammalian cell and maintain in meiotic arrested condition and followed by the inhibition of meiotic maturation [30].

The increasing of oocyte diameter related to the increasing of follicle diameter at WCE 43.2 $\mu \mathrm{g} . \mathrm{mL}^{-1}$ (Fig. 1). It indicates the relationship between follicular diameter and oocyte maturation level characterized by the increasing of oocyte diameter [31]. It happened because there is an acquisition of competence development of oocyte that initiated by interaction between granulosa cells and oocyte through gap junction, su/ch as transfer of small molecules (cAMP, cGMP, RNA, and metabolite) so the oocyte able to regulate oocyte meiotic maturation and reach the final development competence stage [32].

\section{CONCLUSION}

According to our result, WCE exerts its effect on the growth of antral follicle and oocyte based on follicles and oocytes diameter, respectively in a dose-dependent manner after the sixth day in vitro culture of the antral follicle.

\section{ACKNOWLEDGEMENT}

The authors thank the Central Laboratory of Biological Sciences'staff for assistance in conducted research and S. Rahayu, G. Ciptadi for support and facilitate this project.

\section{REFERENCES}

[1] Evans, T.J. 2015. Handbook of toxicology of chemical warfare agents: reproductive toxicity and endocrine disruption of potential chemical warfare agents. Elsevier.

[2] Jones, A.S.K., A. Shikanov. 2019. Follicle development as an orchestrated signaling network in a 3D Organoid. J. Biol Eng. 13(2). doi: 10.1186/s13036-018-0134-3.

[3] Ricardo, J.D.F., L.D.L. Ferreira, J.V.S. Roberto, R.S. Rodrigues. 2018. Control of growth and development of prentral follicle: Insights from In Vitro culture. Anim Reprod. 15(1). 648-659.

[4] Sugino, N. 2005. Reactive Oxygen Specis in ovarian physiology. Reprod. Med Biol. 4. 3144.

[5] Patrick, J.D., D.P. Sally, L. Ulrike. 2012. Roles of Reactive Oxygen Species and antioxidants in ovarian toxycity. Biol. Reprod. 86(2). 2737.

[6] Francesca, C., C. Natascia, D. Danila, T. Simona. 2015. Influence of ROS on ovarian function. New Discoveries in Embriology. 
INTECH open science open mind. doi: 10.5772/61003.

[7] Itoh, T., K. Masayuki, A. Hiroyuki, S. Yutaka, H. Hiroyoshi. 2002. Growth, antrum formation, and estradiol production of bovine preantral follicles cultured in a serum-free medium. Biol. Reprod. 67.10991105.

[8] Harris, A., S. Rahayu, G. Ciptadi. 2014. The morphological measurement of immature oocyte obtained from follicle different size in Indonesia local goat. Int. J Biosci. 4(4). 211-216.

[9] Sugimura, S., D. Richani, R.B. Gilchrist. 2018. Follicular guidance for oocyte developmental competence. Proceedings of the $10^{\text {th }}$ International Ruminant Reproduction Symposium (IRRS2018).

[10] Hardoko, W.L. Gunawan, R. Handayani. 2019. Inhibition activities of water clover (Marcilea crenata) leaf extract on HMG-CoA reduktase enzyme. FaST-Jurnal Sains dan Teknologi. 3(1). 45-57.

[11] Titisari, N., A. Fauzi, A. Adyana, P. Trisunuwati. 2016. the effects of water clover (Marcilea crenata) extract against estrogen, progresteron and uterine histology on rat (Rattus norvegicus). Int. J. PharmTech Res. 9(6). 165-171.

[12] Wahyuningsih, S., G. Ciptadi, K. Pridiawati. 2019. The effect of water clover (Marcilea crenata) extract addition in egg yolk and skim milk extender on frozen goat semen quality. IOP Conference Series: Earth and Environmental Sciences 387012103 . ISTAP.

[13] Trisunuwati, Pratiwi. 2016. The role of leaf water clover (Marsilea crenata) sueeze towards estrogen blood level and uterine histology in rats (Rattus norvegicus). Jurnal Ternak Tropika. 17(2). 1-7.

[14] Sarker, S.D., Z. Latif, A.I. Gray. 2006. An overview of natural product isolation. Natural Product Isolation $2^{\text {nd }}$ Ed. Humana Press Inc. Totowa. 1-26.

[15] Islam, M.S., I. Arihiro, S. Kiyotake, K.N. Hisashi 2017. Isolation and identification of two potential phytotoxic substances from the aquatic fern Marsilea crenata. J. Plant Biol. 60. 75-81.

[16] Ciptadi, G., M.N. Ihsan, S. Rahayu, D.H.K. Widjaya, M. Mudawamah. 2017. A feasibility study of prepubertal and over mature aged local goat in relation to result of In Vitro growth culture to obtain additional M-II oocyte resources. AIP Conference Proceeding. The $8^{\text {th }}$ International Conference on Global Resource Conservation (ICGRC 2017).

[17] Ciptadi, G., S. Rahayu, A.I. Putri, H.N. Karima, A. Budiarto, P.F.P. Susiati, Mudawamah. 2019. Utilization of local goat ovary from slaughterhouse as a material source for in vitro culture, conservation and freezing of oocyte cells. IOP Conf. Series: Earth and Enviromental Science 259.

[18] Silva, G.M., R. Rossetto, R.N. Chaves, A.B.G. Duarte, V.R. Araújo, C. Feltrin, ... J.R. Figueiredo, 2015. In vitro development of secondary follicles from pre-pubertal and adult goats cultured in two-dimensional or three-dimensional systems. Zygote. 23(4). 475-484.

[19] Shimada, M., Y. Yamashita. 2011. The key signaling cascades in granulosa cells during follicular development and ovulation process. J. Mamm. Ova Res. 28. 25-31.

[20] Rosales-Torres, A.M., A.G. Sánchez, C.G. Aguilar. 2012. Review [revisión] follicular development in domestic ruminants [desarrollo folicular en rumiantes domesticos]. Trop. Subtrop.Agroecosystems. 15(1). 147-160.

[21] Martinchik, A.N., V.V. Zubtsov, 2012. Phytoestrogen is properties of flax seed lignans. Vopr Pitan. 81. 61-66.

[22] Cui, J., Y. Shen, R. Li. 2013. Estrogen synthesis and signaling pathways during aging: from periphery to brain. Trends Mol. Med. 19(3). 197-209.

[23] Patel, S., J. Peretz, Y.X. Pan, W.G. Helferich, J.A. Flaws. 2016. Genistein exposure inhibits growth and alters steroidogenesis in adult mouse antral follicles. Toxicol. Appl. Pharmacol. 293. 53-62.

[24] Hashem, N.M., Y.A. Soltan. 2015. Impacts of phytoestrogens on livestock production: a review. $2^{\text {nd }}$ International Conference on the Modern Approaches in Livestock's Production System, Egypt.

[25] Hayashi, S., Y. Yamaguchi. 2008. Estrogen signaling pathway and hormonal therapy. Breast Cancer. 15. 256-261.

[26] Tiemann, U., F. Schneider, J. Vanselow, W. Tomek. 2007. In vitro exposure of porcine granulosa cells to the phytoestrogens genistein and daidzein: Effects on the biosynthesis of reproductive steroid 
hormones. Reprod Toxicol. 24. 317-25. doi: 10.1016/j.reprotox.2007.07.008.

[27] Agarwal, A., S. Gupta, S. Sikka. 2006. The role of free radicals and antioxidants in reproduction. Curr. Opin. Obstet. Gynecol. 18(3). 325-332.

[28] Khazaei, M., A. Faranak. 2017. Reactive Oxygen Species generation and use of antioxidants during In Vitro maturation of oocytes. Int. J. Fertil. Steril. 11(2). 63-70.

[29] Dixon, R.A., D. Ferreira. 2002. Genistein. Phytochemistry. 60. 205-211.

[30] Yoshida, N., K. Mizuno. 2012. Effect of physiological levels of phytoestrogens on mouse oocyte maturation in vitro. Cytotechnology. 64. 241-24.

[31] Mardenli, O., H. Aryan, R. Abdulkarim, M. Al-Mezaid, L. Bogdan. 2017. Effects of follicle size and oocytes diameter on developmental competence and in vitro embryo production of Awassi sheep ewes oocytes. Internasional Educational Applied Scientific Research Journal (IEASRJ). 2(9). 1315.

[32] Li, H.J., M.L., Sutton-McDowall, X. Wang, S. Sugimura, J.G. Thompson, R.B. Gilchrist. 2016. Extending prematuration with cAMP modulators enhances the cumulus contribution to oocyte antioxidant defence and oocyte quality via gap junctions. Hum. Reprod. 31. 810-821. 